\title{
Effectiveness of Isoprothiolane in Preventing Fatty Liver in Dairy Cows
}

\author{
Jun A. Minaguchi ${ }^{1}$, Michisaburo Miura ${ }^{2}$, Shin Oikawa ${ }^{3}$, Fumi Handa ${ }^{1}$, Takuya Haraguchi ${ }^{1}$, \\ Kazuyoshi Miyamoto-Ohno ${ }^{1}$, Yuta Ito ${ }^{1}$, Yoshinao Z. Hosaka, ${ }^{1, \#}$, Hiromi Ueda ${ }^{4}$ and \\ Kazushige Takehana ${ }^{*}, 1$
}

\author{
Laboratory of ${ }^{1}$ Microanatomy, ${ }^{3}$ Herd Health and ${ }^{4}$ Veterinary Anatomy, Rakuno Gakuen University, Ebetsu, Hokkaido \\ 069-8501, Japan; ' Saitama NOSAI, Okegawa branch, Okegawa, Saitama 363-0000, Japan
}

\begin{abstract}
In this study, we evaluated the effectiveness of isoprothiolane in preventing fatty liver in dairy cows. Eight pregnant Holstein cows with high body condition scores were studied. Four cows were administered isoprothiolane via feed at $10 \mathrm{~g} /$ day/head from 21 days before delivery (treated group), while the remaining four cows received no treatment (non-treated group). Liver samples from both groups were obtained 21 days before delivery and 2 days following delivery. No accumulation of lipid droplets in hepatocytes was observed in any cows prior to delivery. Following delivery, the accumulation of lipid droplets in hepatocytes was significantly reduced in the treated group compared to the non-treated group. The number of mitochondria per unit area was also significantly smaller in the treated group than in the non-treated group. Blood and biochemical test results from cows in the treated group showed an increase in mGOT levels and stable intrahepatic ATP production. These results suggest that isoprothiolane treatment intensified beta-oxidation in liver mitochondria, thereby activating the TCA cycle and maintaining intrahepatic ATP production.
\end{abstract}

Keywords: Fatty liver, isoprothiolane, beta-oxidation.

\section{INTRODUCTION}

Fatty liver is one of the most common lipid metabolism disorders in dairy cows. Fatty liver is associated with various periparturient diseases such as ketosis, abomasal displacement, astasia, mastitis, reproductive disorders, and placental retention [1-5].

Cows exhibit significant changes in energy status during the periparturient period. Early lactation is characterized by a negative energy balance, due to both increased energy requirements and decreased feed consumption. To compensate for the shortage in energy, triglycerides (TGs) in the body's fatty tissues are degraded, and resultant non-ester free fatty acids (NEFAs) are mobilized to the liver, where they are metabolized for energy in two major pathways [6]. In one pathway, NEFAs are resynthesized into TGs in hepatocytes. These TGs, together with phospholipids, cholesterol and apolipoprotein B-100, are then transformed to a very low-density lipoprotein (VLDL) subclass that is secreted from the liver to circulating blood. In the other pathway, NEFAs undergo beta-oxidation in the mitochondria and peroxisomes of hepatocytes, and are transformed to acetyl CoA. The TCA cycle then utilizes acetyl CoA to synthesize ATP as an energy source for the body. However, if recruitment of NEFAs exceeds the processing capacity of

*Address correspondence to this author at the Laboratory of Microanatomy, Department of Bioscience, School of Veterinary Medicine, Rakuno Gakuen University, Ebetsu 069-8501, Japan; Tel: +81-11-388-4744; Fax: +81-11388-4854; E-mail: takechan@rakuno.ac.jp

\#Current address: Department of Veterinary Anatomy, Tottori University, Tottori, Japan the liver, VLDL synthesis is down-regulated, and lipid droplets accumulate in hepatocytes - resulting in fatty liver $[7,8]$. This lipid accumulation in hepatocytes also hinders the normal TCA cycle, resulting in an increase in the production of ketone bodies [1], and can physically damage cytoplasmic organelles, including mitochondria $[9,10]$. A decrease in blood vitamin A concentration is thought to be one of the factors responsible for lipid metabolism disorders [4], via the activation of stellate cells and subsequent thickening of the sinusoidal basement membrane [11, 12].

Various isoprothiolane preparations have been used effectively to treat carbon tetrachloride poisoning in heifers [13]. Administration of malotilate, an isoprothiolane analog, has been reported to increase mitochondrial activity and ATP production, and to accelerate the improvement of liver function in rats [14]. While isoprothiolane has been used for a long time in Japan, its mechanism of action remains to be clarified. Therefore, the aim of this study was to determine the effect of isoprothiolane on fatty liver in dairy cows.

\section{MATERIALS AND METHODS}

All animal procedures were approved by the Ethics Committee and the Institutional Animal Use and Care Committee of Rakuno Gakuen University, Ebetsu, Japan.

\section{Animals and Experimental Design}

Eight pregnant Holstein cows with a body condition score of 3.5 or more and their second calving or more were used in this study. The cows were divided equally into isoprothiolane-treated and non-treated groups and the expected date of delivery was determined. Diisopropyl 1,3dithiolan-2-ylidenemalonate (CAS No: 50512-35-1, 
Isoprothiolane, Nihon Nohyaku Co., Ltd., Osaka, Japan) at a dose of $10 \mathrm{~g} /$ day/head in feed was given once daily to the treated group, starting on day 21 of the pre-parturient period (Pre 21) and continuing until calving. The dose of 10 $\mathrm{g} / \mathrm{day} / \mathrm{head}$ was based on the findings of a previous study [14].

Biopsy liver samples were collected from each group on day Pre 21 and day 2 of the post-parturient period (Post 2) following the method described by Mohamed et al. [15]. Blood samples were collected from the jugular vein of each cow prior to daily feeding at days Pre 21, Post 2 and Post 14. Blood and serum samples were stored at $-80^{\circ} \mathrm{C}$ until used for general blood biochemical tests.

\section{Transmission Electron Microscopy}

In preparation for examination by transmission electron microscope (TEM; JEM-1220, JEOL, Tokyo, Japan), biopsied liver samples were cut into small pieces of approximately $1 \mathrm{~mm}^{3}$, immersed in $2.5 \%$ glutaraldehyde in $0.1 \mathrm{M}$ phosphate buffer ( $\mathrm{pH} 7.4$ ), and then post-fixed in $1.0 \%$ osmium tetroxide. The samples were dehydrated and embedded in Quetol 812 (Nissin EM, Tokyo, Japan), sectioned with a diamond knife, and then stained with uranium acetate and lead citrate to facilitate the observation of hepatocytes. Five cells per observation for a total of five fields of each sample were randomly selected in order to calculate the percentage of the hepatocyte occupied by lipid droplets, and the number of mitochondria per unit area (100 $\mu \mathrm{m}^{2}$ ) [16]. For the histochemical TEM analysis, liver samples were cut into small pieces and immersed in a solution of $4.0 \%$ paraformaldehyde- $0.5 \%$ glutaraldehyde in $0.1 \mathrm{M}$ phosphate buffer, with a $\mathrm{pH}$ of 7.4. LR-White (London Resin Company Ltd, Berkshire, England) was used as the embedding medium. We used periodic acidthiocarbohydrazide-silver protein-physical development (PATCH-SP-PD) staining, which reacts with glycosaminoglycans in the basal lamina [17]. The thickness of the basement membrane underlying the sinusoidal endothelium was measured by TEM for each sample [16].

\section{Blood and Biochemical Tests}

A general blood biochemical test and measurement of the serum concentration of vitamin A (VA) were performed by MEDICA JAPAN CO., LTD (Saitama, Japan). Serum glutamic oxaloacetic transaminase (sGOT) and mitochondrial GOT (mGOT) were measured using an automatic analyzer (JCBBM8060, JEOL, Tokyo, Japan). Very low-density lipoprotein
(VLDL) and high-density lipoprotein (HDL) levels were measured using liquid chromatography [18]. Biochemical analysis of intrahepatic ATP production (nmol/g tissue) was performed using an ATP Bioluminescence Assay kit (Roche Diagnostics, Tokyo, Japan) [19].

\section{Statistical Analysis}

The results are expressed as mean \pm SD. Statistical analyses were performed using analysis of covariance (ANCOVA) or Scheffé's test $(\mathrm{p}<0.05)$.

\section{RESULTS}

Morphological analyses of the liver samples are shown in Table 1. On day Pre 21, no lipid droplets were observed in the hepatocytes of cows in either the treated or non-treated groups. By day Post 2, lipid droplets had accumulated in the hepatocytes of cows in both groups (Fig. 1), but the area of the hepatocyte cytoplasm taken up by lipid droplets was significantly smaller in the treated group $(3.6 \% \pm 1.27)$ compared with the non-treated group $(10.5 \% \pm 2.59)$. The number of mitochondria significantly decreased from day Pre 21 to day Post 2 in the treated group, but remained constant in the non-treated group, meaning that, on day Post 2 , liver samples of cows in the treatment group contained significantly fewer mitochondria than those from the nontreated group. The day Pre 21: day Post 2 ratio of mitochondria per $100 \mu^{2}$ was 0.65 in the treated group and 1.03 in the non-treated group. The basement membrane (BM) underlying the sinusoidal endothelium is shown in Fig. (2). The thickness of the BM increased significantly from day Pre 21 to day Post 2 in both groups; however, no significant difference was observed between the two groups. BM thickness increased by $17.5 \%$ between days Pre 21 and Post 2 in the treated group, and by $24.7 \%$ in the non-treated group.

Blood and biochemical data, including VLDL, HDL, sGOT, mGOT, VA, and intrahepatic ATP production, are shown in Table 2. VLDL and HDL levels were high on day Pre 21 but decreased in the post-parturient period in both group, although elevated HDL levels were observed at day Post 14 in the treated group. sGOT levels increased gradually in both groups throughout the study. mGOT levels were higher in the treated group on days Post 2 and Post 14, while VA levels were also higher in the treated group. ATP production was lower in the treated group on day Pre 21, but the levels were similar between the two groups on days Post 2 and Post 14.

Table 1. Morphological Analysis Data of Liver

\begin{tabular}{|c|c|c|c|c|c|c|c|}
\hline \multirow{2}{*}{ Parameters } & \multicolumn{3}{|c|}{ Treated Group } & \multicolumn{3}{|c|}{ Non-Treated Group } & \multirow{2}{*}{ Note } \\
\hline & Pre 21 & Post 2 & *Ratio & Pre 21 & Post 2 & * Ratio & \\
\hline Lipid area $(\%)$ & 0 & $3.6 \pm 1.27$ & 3.6 & 0 & $10.5 \pm 2.59$ & 10.5 & 1 \\
\hline Number of Mitochondoria(/ $\left.100 \mu \mathrm{m}^{2}\right)$ & $38.9 \pm 3.01$ & $25.2 \pm 1.48$ & 0.65 & $37.3 \pm 3.17$ & $38.5 \pm 2.47$ & 1.03 & 2 \\
\hline Thikenes of Basement Membrane (nm) & $58.9 \pm 2.50$ & $69.2 \pm 2.96$ & 1.17 & $55.8 \pm 3.67$ & $69.6 \pm 3.06$ & 1.25 & 3 \\
\hline
\end{tabular}

Mean \pm SD $=\mathrm{P}<0.05$

*: Ratio of between Pre21 to Post 2 in same group

1: Significantly between treated to non-treated group

2: Significantly between pre 21 to post 2 in only treated group

3: Significantly between pre 21 to post 2 in both group 

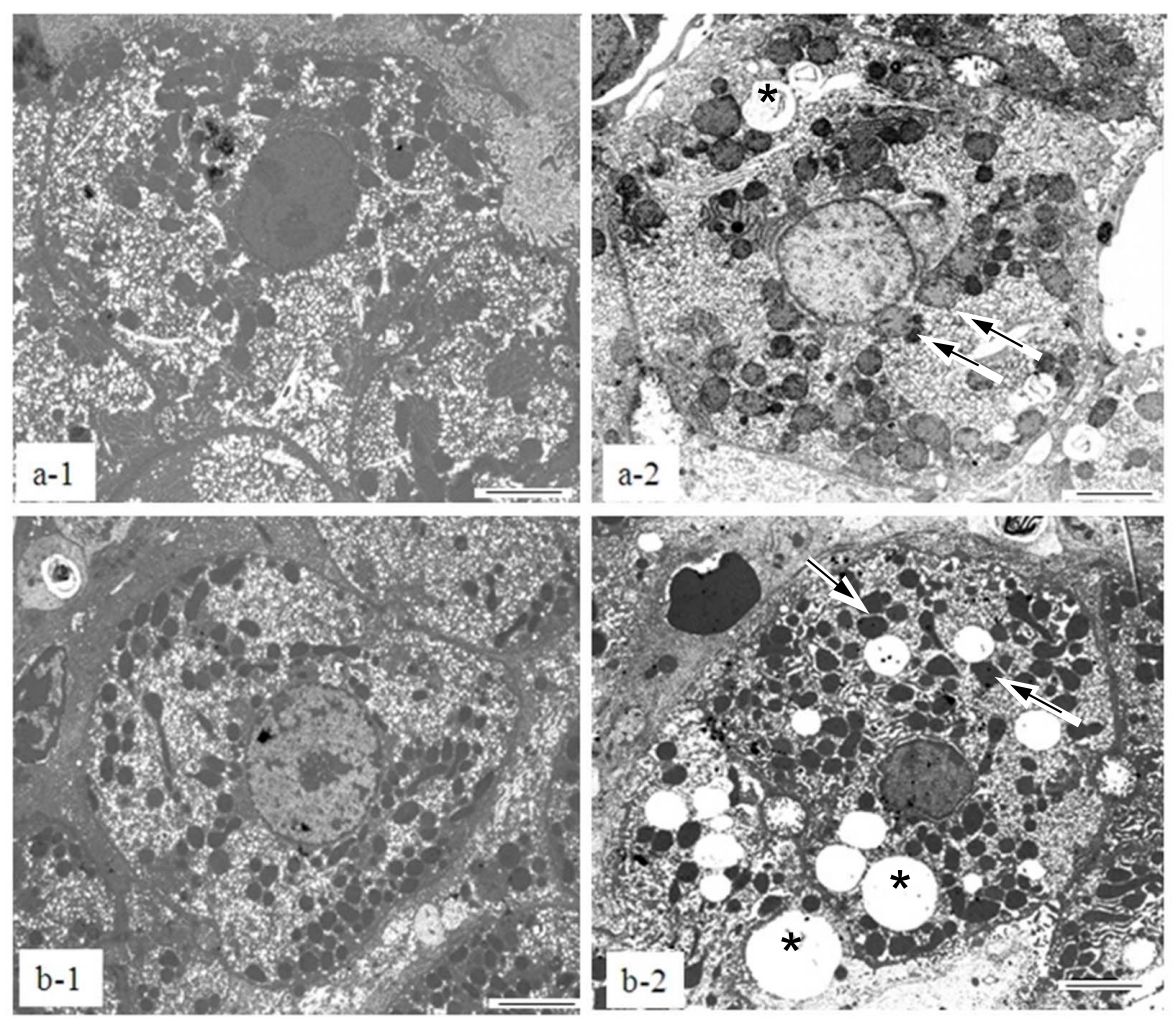

Fig. (1). Change in hepatocyte morphology between the pre-and post-parturient periods. a: treated group, b: non-treated group. 1: day 21 in the pre-parturient period, 2: day 2 in the post-parturient period. Bar $=2 \mathrm{~mm}$. a-1: No lipid droplets evident in the hepatocyte cytoplasm. a-2: Lipid droplets $(*)$ have accumulated in the hepatocyte cytoplasm. The arrows indicate swollen mitochondria. The number of swollen mitochondria in the treated group was much lower compared to that in the non-treated group. b-1: No lipid droplets evident in the hepatocyte cytoplasm. b-2: Lipid droplets (*) have accumulated in the hepatocyte cytoplasm. Abnormally-shaped mitochondria were observed (arrows), caused by pressure from accumulated lipid droplets.

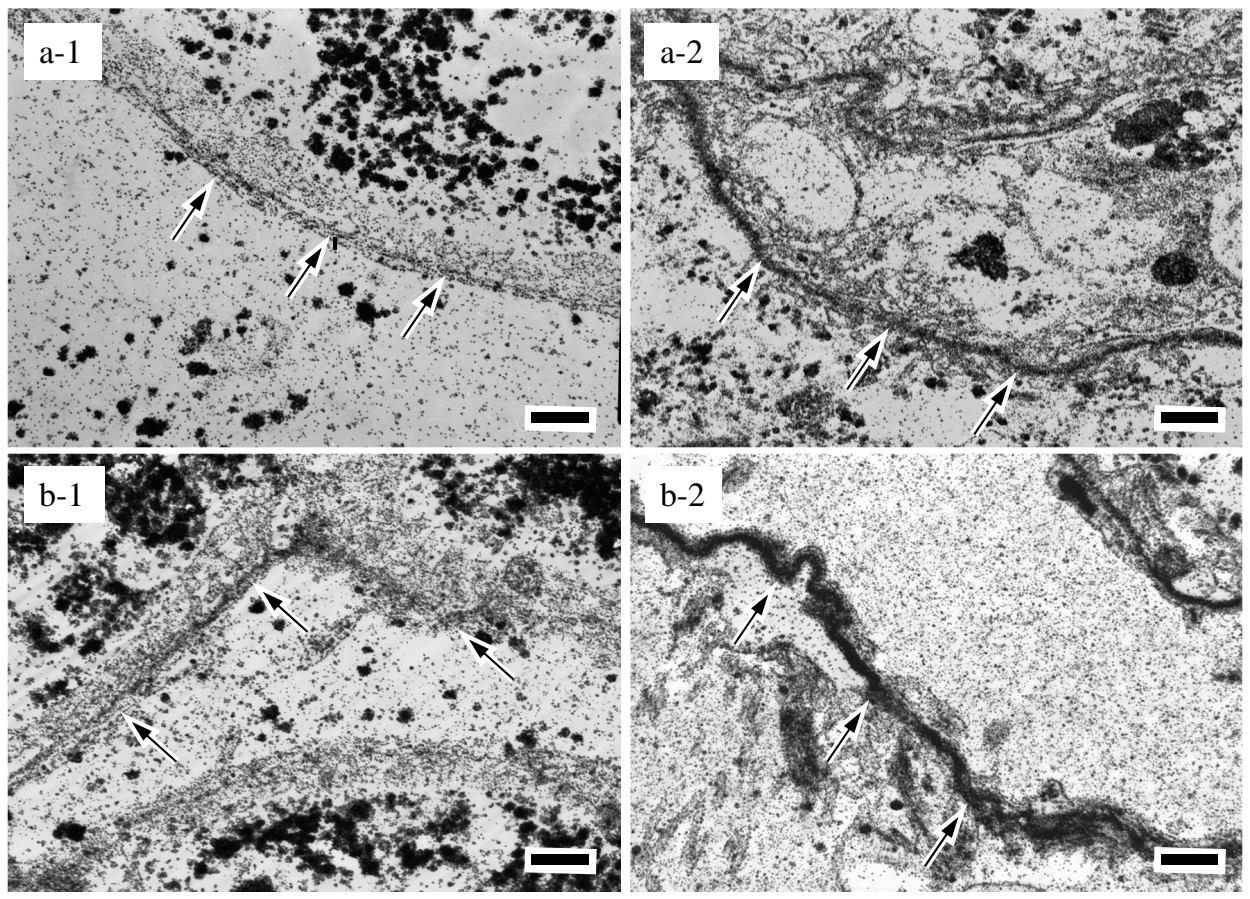

Fig. (2). Ultrastructure of the basement membrane underlying the sinusoidal endothelium, as visualized by periodic acid-thiocarbohydrazidesilver protein-physical development staining. Thickening of basement membranes in the post-parturient period in both treated and nontreated groups was more prominent than that in the pre-parturient period. Bar $=400 \mathrm{~nm}$. a: treated group, b: non-treated group, 1: day 21 in the pre-parturient period, 2: day 2 in the post-parturient period. Arrowheads indicate the basement membrane. 
Table 2. Blood and Biochemical Parameters

\begin{tabular}{|c|c|c|c|c|c|c|}
\hline \multirow{2}{*}{ Parameters } & \multicolumn{3}{|c|}{ Treated Group } & \multicolumn{3}{c|}{ Non-Treated Group } \\
\cline { 2 - 7 } & Pre 21 & Post 2 & Post 14 & Pre 21 & Post 2 & Post 14 \\
\hline \hline VLDL & $16.7 \pm 4.90$ & $0.89 \pm 1.04$ & $1.66 \pm 1.54$ & $20.1 \pm 7.11$ & $0.39 \pm 0.11$ & $1.65 \pm 1.49$ \\
\hline HDL & $87.6 \pm 29.85$ & $74.03 \pm 22.32$ & $122.7 \pm 23.44$ & $78.76 \pm 12.67$ & $65.81 \pm 11.0$ & $80.2 \pm 13.03$ \\
\hline s-GOT(U/1) & $58.30 \pm 8.53$ & $77.30 \pm 15.19$ & $113.5 \pm 18.48$ & $59.4 \pm 21.14$ & $85 \pm 13.28$ & $112.4 \pm 83.4$ \\
\hline mGOT(U/1) & $2.00 \pm 0.82$ & $2.50 \pm 0.58$ & $3.2 \pm 1.89$ & $1.7 \pm 1.10$ & $1.3 \pm 0.84$ & $2.5 \pm 0.55$ \\
\hline Vitamin A(lU/dl) & $34.20 \pm 6.11$ & $22.00 \pm 5.48$ & $25.2 \pm 6.41$ & $21.6 \pm 11.31$ & $10.4 \pm 1.01$ & $14.2 \pm 6.55$ \\
\hline ATP(nmol/g) & $45.20 \pm 10.97$ & $38.40 \pm 8.76$ & $37.5 \pm 9.60$ & $57.2 \pm 10.80$ & $41.2 \pm 6.87$ & $38.2 \pm 4.57$ \\
\hline Mean $\pm \mathrm{SD}=\mathrm{P}<0.05$.
\end{tabular}

\section{DISCUSSION}

Fatty liver is morphologically defined as an accumulation of triglycerides (TGs) in hepatic parenchymal cells. In this study, we found that after delivery, the area within hepatocytes occupied by lipid droplets in the isoprothiolanetreated group $(3.6 \pm 1.27 \%)$ was much smaller than that in the non-treated group $(10.5 \pm 2.59 \%)$, suggesting that isoprothiolane reduced the severity of fatty liver. Furthermore, the number of mitochondria - the organelle responsible for producing ATP from fat metabolism decreased significantly between the pre- and post-parturient periods in the treated group, while in the non-treated group it increased slightly.

This marked difference suggests that isoprothiolane has an effect on the number of mitochondria available to maintain cellular energy levels through ATP production. Therefore, the maintenance of mitochondria in the treated group would promote efficient ATP production, which barely occurred in the non-treated group as indicated by the substantial increase in mitochondrial count. It has previously been reported that administration of malotilate, an isoprothiolane analog, increases mitochondrial activity and ATP production in the rat liver [14]. In dairy cows, isoprothiolane is also likely to stimulate ATP synthesis by inducing the capacity for beta-oxidation of NEFAs in liver mitochondria, as well as their further degradation in the TCA cycle. In this study, ATP recovery levels in hepatocytes of the treated group $(82.9 \%)$ were much higher than that in the non-treated group (66.7\%).

Although sGOT and mGOT enzymes can be generally used as indicators of liver inflammation, mGOT concentration increases only in the presence of necrotic damage to the liver [20]. Interestingly, sGOT levels on day Post2 were lower in the treated group than in the non-treated group, while the opposite was observed for mGOT. It can therefore be speculated that the administration of isoprothiolane in dairy cows with fatty liver increases liver mitochondria activity, and, furthermore, that over-activation of mitochondria can result in increased mGOT levels and a reduction in the number of mitochondria. A substantial number of swollen mitochondria with larger cristae were observed during the post-parturient period in the treated group, a phenomenon that has been attributed to the overactivation of mitochondria [21,22]. Taken together, these results suggest that in the fat metabolic pathway, beta- oxidation in mitochondria was activated by isoprothiolane and ATP production was increased through stimulation of the TCA cycle. This further promoted the metabolism of a substantial amount of fat recruited to the liver, and reduced the accumulation of lipid droplets in hepatocytes.

In the post-parturient period on day 14, the treated group demonstrated an increase in VLDL and a significant increase in HDL compared with the non-treated group. These results suggest that increased mitochondrial function facilitates overall fat metabolism.

It has been reported that cows with fatty liver and low serum VA concentration exhibit a reduction in the size of cytoplasmic lipid droplets in stellate cells, and thickening of the BM underlying the sinusoidal endothelium [23]. In this study post-parturient VA concentration dropped significantly in the non-treated group, and the VA concentration in the treated group remained higher than in the non-treated group throughout the study period. In addition, the treated group showed less thickening of the BM after delivery compared with the non-treated group. Thus, isoprothiolane may have the potential to increase the secretion of VA from the liver, allowing serum VA levels to be maintained and resulting in reduced thickening of the BM.

Based on the difference between the treated and nontreated groups demonstrated by this study in terms of morphological changes and the degree of hepatic fat accumulation, it can be hypothesized that treatment with isoprothiolane stimulates fatty acid catabolism in the livers of dairy cows and may therefore be effectively used for the prevention of fatty liver in these animals.

\section{CONFLICT OF INTEREST}

The authors confirm that this article content has no conflicts of interest.

\section{ACKNOWLEDGEMENTS}

We are grateful to Dr. Watanabe (Shinshu University) and Dr. Nagayasu (Menicon) for discussion and assistance.

\section{REFERENCES}

[1] Brumby PE, Anderson M, Tuckley B, Storry JE, Hibbit KG. Lipid metabolism in the cow during starvation-induced ketosis. Biochem J 1975; 146: 609-15.

[2] Nakagawa-Ueta H, Katoh N. Reduction in serum lecithin: Cholesterol acyltransferase activity prior to the occurrence of ketosis and milk fever in cows. J Vet Med Sci 2000; 62: 1263-7. 
[3] Oikawa S, Katoh N. Reduced concentrations of apolipoproteins B100 and A-I in serum from cows with retained placenta. Can J Vet Res 1997; 61(4): 312-4.

[4] Oikawa S, Katoh N, Kawawa F, Ono Y. Decreased serum apolipoprotein B-100 and A-I concentrations in cows with ketosis and left displacement of the abomasum. Am J Vet Res 1997; 58: 121-5.

[5] Reid IM. An ultrastructural and morphometric study of the liver of the lactating cow in starvation ketosis. Exp Mol Pathol 1973; 18: 316-30.

[6] Herdt TH. Ruminant adaptation to negative energy balance. Influences on the etiology of ketosis and fatty liver. Vet Clin North Am Food Anim Pract 2000; 16: 215-30.

[7] Herdt TH. Fatty liver in dairy cows. Vet Clin North Am Food Anim Pract 1988; 4: 269-87.

[8] Vogel F, Bornhövd C, Neupert W, Reichert AS. Dynamic subcompartmentalization of the mitochondrial inner membrane. J Cell Biol 2006; 175: 237-47.

[9] Reid IM, Collins RA. The pathology of post-parturient fatty liver in high-yielding dairy cows. Invest Cell Pathol 1980; 3: 237-49.

[10] Senoo H, Imai K, Matano Y, Sato M. Hepatic stellate cells: Molecular mechanisms in the reversible regulation of morphology, proliferation and collagen metabolism in hepatic stellate cells by the three-dimensional structure of the extracellular matrix. J Gastroenterol Hepatol 1998; 13: S19-32.

[11] Davis BH, Rapp UR, Davidson NO. Retinoic acid transforming growth factor $\mathrm{b}$ differentially inhibits platelet-derived-growthfactor-intensified Ito-cell activation. Biochem J 1991; 278: 43-7.

[12] Studer VA, Grummer RR, Bertics SJ, Reynolds CK. Effect of prepartum propylene glycol administration on periparturient fatty liver in dairy cows. J Dairy Sci 1993; 76: 2931-9.

[13] Nagasawa S, Yamanaka H, Motoi Y, Ishikawa T, Takizawa T. Effect of isoprothiolane on hepatic lesions of heifer caused by carbon tetrachloride. Nippon Juigaku Zasshi 1989; 51: 284-93.
[14] Niwano Y, Konaka S, Uchida M, Sugimoto T. Activation of mitochondrial functions by malotilate in relation to accelerated liver regeneration in partially hepatectomized rats. Jpn J Pharmacol 1986; 42: 525-9.

[15] Mohamed T, Oikawa S, Kurosawa T, et al. Focal fatty liver in a heifer: utility of ultrasonography in diagnosis. J Vet Med Sci 2004; 66: 341-4.

[16] Higashi $\mathrm{N}$, Ueda $\mathrm{H}$, Yamada $\mathrm{O}$, et al. Micromorphological characteristics of hepatic sinusoidal endothelial cells and their basal laminae in five different animal species. Okajimas Folia Anat Jpn 2002; 79: 135-42.

[17] Yamada K. Histochemistry of carbohydrates as performed by physical development procedures. Histochem J 1993; 25: 95-106.

[18] Vazquez-Añon M, Bertics S, Luck M, Grummer RR, Pinheiro J. Peripartum liver triglyceride and plasma metabolites in dairy cows. J Dairy Sci 1994; 77: 1521-8.

[19] Li J, Aroutcheva AA, Faro S, Chikindas ML. Mode of action of lactocin 160, a bacteriocin from vaginal Lactobacillus rhamnosus. Infect Dis Obstet Gynecol 2005; 13: 135-40.

[20] Gerloff BJ, Herdt TH, Emery RS. Relationship of hepatic lipidosis to health and performance in dairy cattle. J Am Vet Med Assoc 1986; 188: 845-50.

[21] Braunstein AE. Amino group transfer, L-Asparte: 2-oxoglutarate aminitransferase. In: Boyer, PD, Krebs ED, Eds. The enzymes. $3^{\text {rd }}$ ed. New York: Academic Press 1973; pp. 393-8.

[22] Perkins GA, Ellisman MH, Fox DA. Three-dimensional analysis of mouse rod and cone mitochondrial cristae architecture: bioenergetic and functional implications. Mol Vis 2003; 9: 60-73.

[23] Ogawa M, Imai K, Oikawa S, Hosaka Y, Ueda H, Takehana K. Relationship between Serum vitamin-A concentration and hepatic stellate cells in cows with fatty-liver disease. J Jpn Vet Med Assoc 2004; 57: 639-43. (in Japanese). 